\title{
Correction to: Poles and residues of standard $L$-functions attached to Siegel modular forms
}

\section{Shin-ichiro Mizumoto ${ }^{1}$}

Published online: 18 August 2020

(c) Springer-Verlag GmbH Germany, part of Springer Nature 2020

\section{Correction to: Math. Ann. 289:589-612 (1991) https://doi.org/10.1007/BF01446591}

Abstract Correction to my paper on the poles of standard $L$-functions attached to Siegel modular forms.

\section{Mathematics Subject Classification 11F46 • 11F66}

In the second corollary of Theorem 3 of the original paper [5, p. 601], I made a statement (without proof) on the orders of possible poles of the completed standard $L$-function $\Lambda(s, f, \underline{\mathrm{St}})$ attached to a Siegel modular form $f$ of weight $k$ and degree $n$ in case $k<n$. It asserts in particular that the orders of poles are at most two, but this is not the case.

This error was pointed out by a mail from Professor Chenevier on October 27, 2018 (for details, see the remark below). After that I checked the paper again and noticed that I had made some mistakes in deducing the claim.

To state the corrected version the following notation will be used. For $n, k \in \mathbf{Z}_{>0}$ let $S_{k}^{n}$ be the space of holomorphic cusp forms of weight $k$ for

$$
\Gamma^{(n)}:=\operatorname{Sp}(n, \mathbf{Z})=\operatorname{Sp}_{2 n}(\mathbf{Z}) .
$$

Let $f \in S_{k}^{n}$ be a Hecke eigenform and $L(s, f, \underline{\mathrm{St}})$ be the standard $L$-function attached to $f$. Take $\varepsilon, v \in\{0,1\}$ such that $n \equiv \varepsilon(\bmod 2)$ and $k \equiv v(\bmod 2)$. Let

$$
\Gamma_{\mathbf{R}}(s):=\pi^{-\frac{s}{2}} \Gamma\left(\frac{s}{2}\right), \quad \Gamma_{\mathbf{C}}(s):=2(2 \pi)^{-s} \Gamma(s)
$$

\section{Communicated by Wei Zhang.}

The original article can be found online at https://doi.org/10.1007/BF01446591.

Shin-ichiro Mizumoto

mizumoto@math.titech.ac.jp

1 Department of Mathematics, Tokyo Institute of Technology, Oo-okayama, Meguro-ku, Tokyo 152-8551, Japan 
and

$$
\Lambda(s, f, \underline{\mathrm{St}}):=\Gamma_{\mathbf{R}}(s+\varepsilon) \prod_{j=1}^{n} \Gamma_{\mathbf{C}}(s+k-j) L(s, f, \underline{\mathrm{St}}) .
$$

By Böcherer [1], $\Lambda(s, f, \underline{\mathrm{St}})$ has a meromorphic continuation to the whole $s$-plane and is invariant under the substitution $s \mapsto 1-s$. The symbol $\operatorname{ord}_{s=c}$ stands for the order of zero of a meromorphic function at $s=c$. The largest integer $\leq x$ is denoted by $[x]$.

Now we state the correction. The second corollary of Theorem 3 in [5] should be replaced by the following

Proposition 1 Suppose $k<n$. Then the possible poles of $\Lambda(s, f, \underline{\mathrm{St}})$ are contained in $[-n+k-v, n-k+v+1] \cap \mathbf{Z}$ and for every $m \in[1, n-k+v+1] \cap \mathbf{Z}$ we have

$$
\operatorname{ord}_{s=m} \Lambda(s, f, \underline{\mathrm{St}}) \geq\left[\frac{m-n}{2}\right]+\frac{k-v}{2}-1
$$

\section{Proof Put}

$$
\Gamma_{n}(s):=\prod_{j=1}^{n} \Gamma\left(s-\frac{j-1}{2}\right), \quad C_{n}(s):=\prod_{j=1}^{n}\left(s+\frac{j-1}{2}\right)=\frac{\Gamma_{n}\left(s+\frac{n+1}{2}\right)}{\Gamma_{n}\left(s+\frac{n-1}{2}\right)},
$$

and

$$
\mu(n, k, s):=(-1)^{\frac{n k}{2}} 2^{\frac{n^{2}+3 n}{2}-2 n s-n k+1} \pi \frac{n(n+1)}{2} \frac{\Gamma_{n}\left(s+k-\frac{n+1}{2}\right)}{\Gamma_{n}(s+k)} .
$$

For $m \in \mathbf{Z}_{>0}$ let $\mathfrak{H}_{m}$ be the Siegel upper half space of degree $m$. For $w \in 2 \mathbf{Z}_{\geq 0}, z \in \mathfrak{H}_{m}$ and $s \in \mathbf{C}$ with $\operatorname{Re}(w+2 s)>m+1$ let

$$
E_{w}^{(m)}(z, s):=\operatorname{det}(\operatorname{Im}(z))^{s} \sum_{\{c, d\}} \operatorname{det}(c z+d)^{-w}|\operatorname{det}(c z+d)|^{-2 s}
$$

be the nonholomorphic Eisenstein series of weight $w$ for $\Gamma^{(m)}$. Here $\left(\begin{array}{ll}* & * \\ c & d\end{array}\right)$ runs

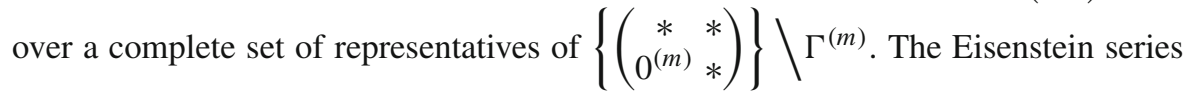
$E_{w}^{(m)}(z, s)$ has a meromorphic continuation to the whole $s$-plane. By Böcherer [1, p. 157], 


$$
\begin{aligned}
L(s, f, \underline{\mathrm{St}}) f(Z) & \left(2^{n} C_{n}\left(1-\frac{s+k+v+n}{2}\right)\right)^{-\delta\left(\frac{v-1}{2}\right)} \mu\left(n, k, \frac{s-k+v+n}{2}\right)^{-1} \\
& \cdot \zeta(s+n) \prod_{j=0}^{n-1} \zeta(2 s+2 j) \\
& \cdot\left(f(W),\left(\left(D^{v} E_{k-v}^{(2 n)}\right)\left(*, \frac{\bar{s}-k+v+n}{2}\right)\right)\left(\begin{array}{cc}
-\bar{Z} & 0 \\
0 & W
\end{array}\right)\right)
\end{aligned}
$$

for every $Z \in \mathfrak{H}_{n}$. Here $\delta(x)=1$ or 0 according as $x \in \mathbf{Z}$ or not; for

$$
\mathcal{Z}=\left(\begin{array}{cc}
Z & U \\
{ }^{t} U & W
\end{array}\right) \in \mathfrak{H}_{2 n} \quad \text { with } Z, W \in \mathfrak{H}_{n} \text { and } U=\left(u_{i j}\right)
$$

the differential operator $D$ is defined by

$$
D:=\operatorname{det}\left(\frac{\partial}{\partial u_{i j}}\right)_{1 \leq i, j \leq n}
$$

By the functional equation of $\Lambda(s, f, \underline{\mathrm{St}})$ we restrict our attention to the poles of the functions in (a) in the right half plane $\operatorname{Re}(s)>0$. In the following let $g_{j}(s)(j=$ $1,2, \ldots)$ be some functions which are holomorphic and nonzero in $\operatorname{Re}(s)>0$. Note that

$$
C_{n}\left(1-\frac{s+k+v+n}{2}\right)=(-1)^{n} C_{n}\left(\frac{s+k+v-1}{2}\right)=g_{1}(s)
$$

and

$$
\mu\left(n, k, \frac{s-k+v+n}{2}\right)^{-1}=g_{2}(s) \cdot \Gamma_{n}\left(\frac{s+k+v-1}{2}\right)^{-1} .
$$

For $N \in 2 \mathbf{Z}_{>0}$ and $z \in \mathfrak{H}_{m}$ let $E^{*}\left(z, s ; w, \operatorname{triv}_{N}, N\right)$ be the Eisenstein series of level $N$ defined in Feit [3, p. 11], with triv ${ }_{N}$ being the trivial Dirichlet character modulo $N$. By Shimura [8, Proposition 2.1],

$$
E_{w}^{(m)}(z, s)=\operatorname{Tr}_{1}^{N}\left(\left.E^{*}\left(z, s ; w, \operatorname{triv}_{N}, N\right)\right|_{w} \eta_{m}\right)
$$

where $\eta_{m}:=\left(\begin{array}{cc}0 & 1_{m} \\ -1_{m} & 0\end{array}\right)$ and $\operatorname{Tr}_{1}^{N}$ is the trace map from $\Gamma_{0}^{(m)}(N)$ to $\Gamma^{(m)}$ defined as in $[5$, p. 592]. Rewriting this in the notation of Feit [3, p. 48], we have 


$$
\begin{aligned}
& E_{k-v}^{(2 n)}\left(\mathcal{Z}^{(2 n)}, \frac{s-k+v+n}{2}\right) \\
& \quad=g_{3}(s) \zeta(2 s)^{-1} \operatorname{Tr}_{1}^{N}\left(\left.D\left(\mathcal{Z}, \frac{s-k+v+n}{2} ; k-v, \operatorname{triv}_{N}, N\right)\right|_{k-v} \eta_{2 n}\right) .
\end{aligned}
$$

Hence by (a), for every $c \in \mathbf{C}$ with $\operatorname{Re}(c)>0$ we have

$$
\operatorname{ord}_{s=c} L(s, f, \underline{\mathrm{St}}) \geq \inf _{\mathcal{Z} \in \mathfrak{H}_{2 n}} \operatorname{ord}_{s=c} \frac{D\left(\mathcal{Z}, \frac{s-k+v+n}{2} ; k-v, \operatorname{triv}_{N}, N\right)}{\Gamma_{n}\left(\frac{s+k+v-1}{2}\right)},
$$

where $N \in 2 \mathbf{Z}_{>0}$ is arbitrary. By [3, p. 49], every possible pole of

$$
D\left(\mathcal{Z}^{(2 n)}, \frac{s-k+v+n}{2} ; k-v, \operatorname{triv}_{N}, N\right)
$$

in $\operatorname{Re}(s)>0$ is simple and contained in $[1, n-k+v+1] \cap \mathbf{Z}$. In particular, the possible poles of $\Lambda(s, f, \underline{\mathrm{St}})$ are contained in $\mathbf{Z}$. Now let $m \in \mathbf{Z}_{>0}$. Then

$$
\begin{aligned}
\operatorname{ord}_{s}=m & \Gamma_{n}\left(\frac{s+k+v-1}{2}\right) \\
\quad & =-\delta(m \leq n-k-v)\left(\left[\frac{n-m}{2}\right]-\frac{k+v}{2}+1\right),
\end{aligned}
$$

where $\delta(C):=1$ or 0 according as the condition $C$ is satisfied or not. Hence from (b) it follows that

$$
\begin{aligned}
\operatorname{ord}_{s=m} L(s, f, \underline{\mathrm{St}}) \geq & \delta(m \leq n-k-v)\left(\left[\frac{n-m}{2}\right]-\frac{k+v}{2}+1\right) \\
& -\delta(m \leq n-k+v+1) .
\end{aligned}
$$

On the gamma factor of $\Lambda(s, f, \underline{\mathrm{St}})$ we have

$$
\operatorname{ord}_{s=m} \Gamma_{\mathbf{R}}(s+\varepsilon) \prod_{j=1}^{n} \Gamma_{\mathbf{C}}(s+k-j)=\delta(m \leq n-k)(m-n+k-1) .
$$

From (c) and (d) the assertion of Proposition 1 follows.

Remark (1) Proposition 1 follows also from Shimura [9, Theorem 6.1], which is much more extensive.

(2) By the above-mentioned mail from Professor Chenevier I was informed of the following: Let $F \in S_{13}^{24}$ be the Hecke eigenform discovered by Freitag [4], Satz 5.2. Then by the theory of theta lift for the orthogonal group $O(24)$ of ChenevierLannes [2] combined with the Eichler commutation relations of Rallis [7], the equality 


$$
L(s, F, \underline{\mathrm{St}})=\prod_{i=0}^{11} L(s+11-i, \Delta) \prod_{j=0}^{24} \zeta(s+12-j)
$$

holds, where $L(s, \Delta)$ is the Hecke $L$-function attached to Ramanujan's $\Delta \in S_{12}^{1}$ which satisfies a functional equation under $s \mapsto 12-s$. This implies in particular that $\Lambda(s, F, \underline{\mathrm{St}})$ has a pole of order $7-[m / 2]$ at every $s=m \in[1,13] \cap \mathbf{Z}$. Note that Proposition 1 gives the best possible bound in this case.

Professor Chenevier pointed out also that the Langlands standard gamma factor for $L(s, f, \underline{\mathrm{St}})$ in case $k<n$ is

$$
\gamma_{k}^{(n)}(s):=\Gamma_{\mathbf{R}}(s+v) \prod_{i=1}^{k} \Gamma_{\mathbf{C}}(s+k-i) \prod_{j=1}^{n-k} \Gamma_{\mathbf{R}}(s+v+j) \Gamma_{\mathbf{R}}(s+v-j)
$$

according to Moeglin-Renard [6], Proposition 9.1. Hence let

$$
\Lambda^{*}(s, f, \underline{\mathrm{St}}):=\gamma_{k}^{(n)}(s) L(s, f, \underline{\mathrm{St}}),
$$

which also satisfies

$$
\Lambda^{*}(s, f, \underline{\mathrm{St}})=\Lambda^{*}(1-s, f, \underline{\mathrm{St}}) .
$$

For every $m \in \mathbf{Z}_{>0}$ we have

$$
\operatorname{ord}_{s=m} \gamma_{k}^{(n)}(s)=-\delta(m \leq n-k-v)\left(\left[\frac{n-m}{2}\right]-\frac{k+v}{2}+1\right) .
$$

Hence (c), (e), and (f) imply that in terms of $\Lambda^{*}(s, f, \underline{\mathrm{St}})$ Proposition 1 takes the following form:

Proposition 2 For any Hecke eigenform $f \in S_{k}^{n}$ with $k<n$, every possible pole of $\Lambda^{*}(s, f, \underline{\mathrm{St}})$ is simple and contained in $[-n+k-v, n-k+v+1] \cap \mathbf{Z}$.

Acknowledgements The author would like to thank Professor Chenevier for valuable advice.

\section{References}

1. Böcherer, S.: Über die Funktionalgleichung automorpher L-Funktionen zur Siegelschen Modulgruppe. J. Reine Angew. Math. 362, 146-168 (1985)

2. Chenevier, G., Lannes, J.: Automorphic Forms and Even Unimodular Lattices. Ergebnisse der Mathematik und ihrer Grenzgebiete. 3. Folge./A Series of Modern Surveys in Mathematics (Book 69). Springer, Berlin (2019)

3. Feit, P.: Poles and residues of Eisenstein series for symplectic and unitary groups. Mem. Am. Math. Soc. 61(346) (1986)

4. Freitag, E.: Die Wirkung von Heckeoperatoren auf Thetareihen mit harmonischen Koeffizienten. Math. Ann. 258, 419-440 (1982) 
5. Mizumoto, S.: Poles and residues of standard L-functions attached to Siegel modular forms. Math. Ann. 289, 589-612 (1991)

6. Moeglin, C., Renard, D.: Sur les paquets d'Arthur de $\operatorname{Sp}(2 n, \mathbf{R})$ contenant des modules unitaires de plus haut poids, scalaires. arXiv:1802.04611v4

7. Rallis, S.: Langlands functoriality and the Weil representation. Am. J. Math. 104, 469-515 (1982)

8. Shimura, G.: On Eisenstein series. Duke Math. J. 50, 417-476 (1983)

9. Shimura, G.: Eisenstein series and zeta functions on symplectic groups. Invent. Math. 119, 539-584 (1995)

Publisher's Note Springer Nature remains neutral with regard to jurisdictional claims in published maps and institutional affiliations. 